\title{
Dark Current Analysis of Mid-Wave Quantum Dots- in-a-Well Photodetectors Monolithically Grown on Silicon Substrate
}

\author{
Wei Chen ${ }^{1}$, Zhuo Deng ${ }^{1}$, Jian Huang ${ }^{1}$, Baile Chen ${ }^{1}$, Huiyun Liu $^{2}$, Jiang Wu ${ }^{2}$ \\ ${ }^{1}$ School of Information Science and Technology, ShanghaiTech University, Shanghai, 201210, China \\ ${ }^{2}$ Department of Electronic and Electrical Engineering, University College London, London WC1E 7JE, UK
}

\begin{abstract}
In this work, we analyzed the dark current performance of the InAs/InGaAs/GaAs quantum dots-in-a-well (DWELL) mid-wave infrared photodetectors monolithically grown on silicon substrate.
\end{abstract}

Keywords-quantum dots-in-a-well; mid-wave infrared photodetectors, silicon photonics; dark current; dark noise

\section{INTRODUCTION}

Mid-infrared (MIR) silicon photonics have recently attracted extensive attention due to the integrated solution for gas sensing, defense and medical applications with minimal cost. As the key component of MIR silicon photonics systems, it would be desirable to monolithically grow MIR photodetectors on silicon substrate along with other active and passive MIR devices[1]. Moreover, by monolithic growth of infrared photodetector materials on large $\mathrm{Si}$ substrate, one could substantially reduce the cost of focal plane array (FPA). Our group, for the first time, demonstrated the mid-wave infrared InAs/InGaAs/GaAs dots-in-a-well structure grown in $\mathrm{Si}$ substrate, which shows great potential for future MIR silicon photonics and low cost FPA applications[2]. In this paper, we use the sequential coupling model to investigate the dark current performance of the devices.

\section{DEVICE Straucture AND FABRICATIONS}

The sample structure is shown in Fig. 1. The InAs/InGaAs/GaAs DWELL structures were grown on $\mathrm{Si}(100)$ substrate using a Veeco Gen-930 molecular beam epitaxy system. The Si substrate has $4^{\circ}$ offcut towards the [011] direction. Firstly, a 1000 nm n-type GaAs layer was grown on $\mathrm{Si}$ substrate as buffer layer. Four repeats of dislocation filter layers (DFLs) of InGaAs/GaAs superlattices (SLSs) were then grown to inhibit the propagation of dislocation. After depositing four repeats of the DFLs, the threading dislocation density can reach a level of $\sim 3 \times 10^{6} / \mathrm{cm}^{2}$. After growing the DFLs layer, the $500 \mathrm{~nm}$ n-type GaAs bottom contact layer with $80 \mathrm{~nm}$ undoped GaAs spacer layer was grown. The active region of DWELL structure includes 20 periods of $50 \mathrm{~nm}$ undoped GaAs spacer layer, the InAs QD (Si-doped, 2 electrons/dot) layer sandwiched between two InGaAs quantum wells, with thickness of $6 \mathrm{~nm}$ and $2 \mathrm{~nm}$ respectively. Finally, a $30 \mathrm{~nm}$ undoped GaAs spacer layer and 300 nm n-type GaAs layer were grown as top contact.

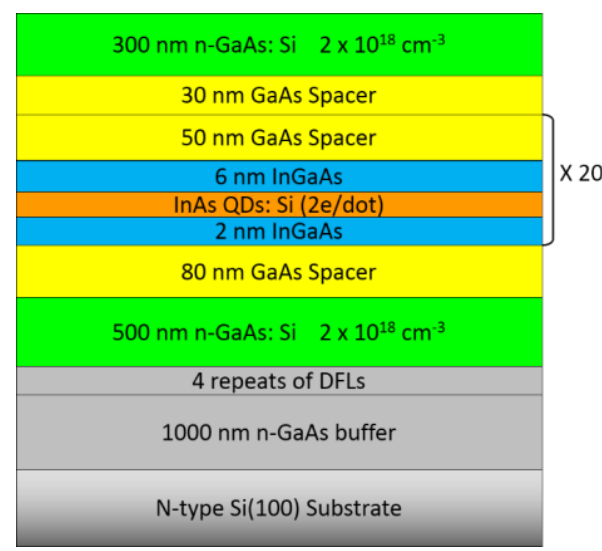

Fig. 1. Schematic structure of the InAs/InGaAs/GaAs DWELL structure.

After the material growth, the DWELL sample was fabricated into a set of unpassivated mesa-isolated circular devices with nominal diameters ranging from $20 \mu \mathrm{m}$ to $500 \mu \mathrm{m}$ using standard UV photolithography and wet chemical etching. $\mathrm{Ti} / \mathrm{Au}(50 \mathrm{~nm} / 300 \mathrm{~nm})$ were deposited at the top and bottom $\mathrm{n}+$ doped GaAs layers for metal contact.

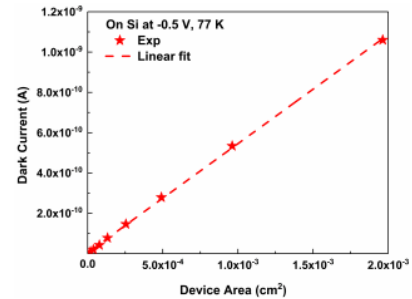

(a)

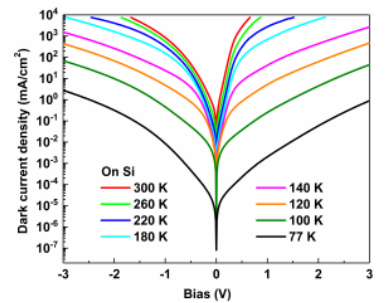

(b)
Figure 2(a) Current v.s device area with bias of $-0.5 \mathrm{~V}$ at $77 \mathrm{~K}$. (b) Current density of the device from $77 \mathrm{~K}$ to $300 \mathrm{~K}$.

\section{DARK CURRENT ANALYSIS}

The dark current-voltage $(I-V)$ characteristics of the DWELL photodetector devices were measured with semiconductor device analyzer in the low temperature probe station from 77 to $300 \mathrm{~K}$. The dark current scales linearly with device area as shown in Figure 2 (a) 
which indicates that the dark current is dominant by the bulk current instead of surface leakage. The dark current density of the device is shown in Figure 2(b). The device shows dark current density of $8.5 \times$ $10^{-3} \mathrm{~mA} / \mathrm{cm}^{2}$ at $77 \mathrm{~K}$ and increase to $0.84 \mathrm{~A} / \mathrm{cm}^{2}$ at $300 \mathrm{~K}$.

To better understand the dark current properties of the DWELL structure, the sequential coupling model is used to investigate and fit the experimental data. The sequential coupling model was first proposed by L.Lin etc.[3], which include the two activation energy. The total activation energy is the sum of the microscale activation energy $E_{a, \text { micro }}$ and nanoscale activation energy $E_{a, \text { nano }}$ respectively as illustrated in equation 1 and Figure 3 . The $E_{a \text {,micro }}$ is the microscale electron transport activation energy, which equals to the energy difference between the top of the AlGaAs barrier layer and the Fermi level $\left(\mathrm{E}_{\mathrm{F}}\right), E_{0, \text { micro }}$ is the microscale electron transport activation energy at zero bias, $E_{0}$ is the fitting parameter, which represents the change rate of the $E_{a \text {,micro }}$ with bias. $E_{a, \text { nano }}$ is the nanoscale electron transport activation energy, which equals to the excitation energy of QDs. $E_{0, \text { nano }}$ is the nanoscale electron transport activation energy at zero bias, $\beta$ is also the fitting parameter, which represents the change rate of the $E_{a, \text { nano }}$ with electrical field. $E$ is the applied electric field, which equals to the ratio of the external bias voltage divided by the intrinsic absorption region thickness of the device.

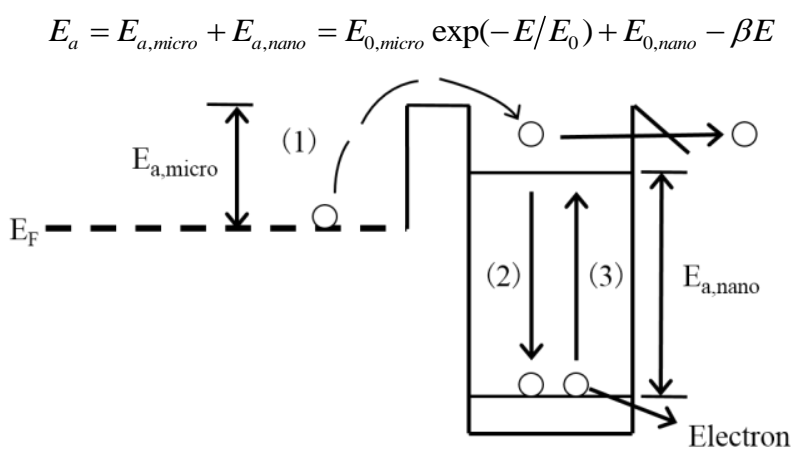

Figure 3 Schematics diagram of the sequential coupling dark current model

With assumption that uniform electrical field across the DWELL active region, the dark current data along with the fitting results were shown in the Figure 4. All the fitting parameters are shown in Table I for both negative and positive bias from $77 \mathrm{~K}$ to $180 \mathrm{~K}$. It is shown in Table I that the fitting parameters vary as the bias polarization changes. That is mainly due to the asymmetric geometry of the quantum dot growth, and carriers experience different energy barriers as they travel towards to the top or bottom contacts. As indicated in Figure 4, the model fits very well from $77 \mathrm{~K}$ to $180 \mathrm{~K}$ as bias increasing from \pm 1 to \pm 3 . However, it is also noted that the fitting curves deviate from the experimental data at small bias. The reason is explained as below. Given the previously photoluminescence results, it is shown that the QDs distribution is inhomogeneous, in other word, there are some dots with different sizes in the structure.
Some inter-dots carrier transfer process from smaller dots to larger dots happens as applied bias increase. It is expected dots with different sizes will have different values of nanoscale activation energy, the current model with one single nanoscale activation energy does not take account all these affects. That is why there is some deviation between experimental data and fitting results at small bias. Therefore, some future work to include the additional nanoscale activation may be needed to improve the model itself.

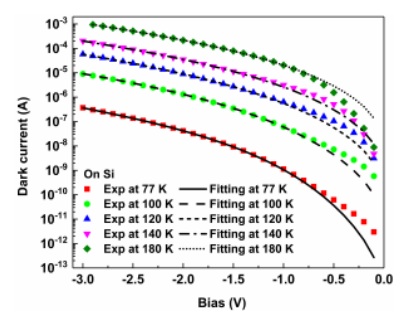

(a)

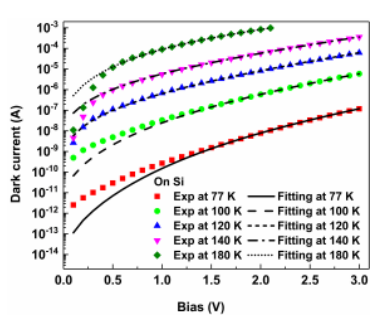

(b)
Figure 4 (a) dark current fitting result along with experimental data at negative bias. (b) dark current fitting result along with experimental data at positive bias.

TABLE I: THE FITTING PARAMETERS OF THE DWELL PHOTODETECTOR ON SI AT VARIOUS TEMPERATURES WITH BOTH NEGATIVE AND POSITIVE BIAS

\begin{tabular}{|c|c|c|c|c|}
\hline $\mathrm{T}$ & $E_{0, \text { micro }}(\mathrm{meV})$ & $E_{0}(\mathrm{kV} / \mathrm{cm})$ & $E_{0, \text { nano }}(\mathrm{meV})$ & $\beta$ (meV.cm/kV) \\
\hline $77 \mathrm{~K}$ & $57.6 / 49.52$ & $7074.2 / 9962.2$ & $141.1 / 152.8$ & $1.22 / 1.51$ \\
\hline $100 \mathrm{~K}$ & $56.83 / 41.19$ & $9181.4 / 9457.9$ & $149.98 / 168.44$ & $1.28 / 1.86$ \\
\hline $120 \mathrm{~K}$ & $51.4 / 22.76$ & $9132.5 / 9048.6$ & $167.79 / 183.15$ & $1.65 / 2.24$ \\
\hline $140 \mathrm{~K}$ & $53.09 / 21.64$ & $8904.1 / 8822.8$ & $182.94 / 190.45$ & $1.87 / 2.39$ \\
\hline $180 \mathrm{~K}$ & $55.66 / 49.2$ & $8390.4 / 5878.2$ & $215.07 / 202.39$ & $2.35 / 2.54$ \\
\hline
\end{tabular}

\section{CONCLUSION}

In this paper, the sequential coupling model is presented to analyze the dark current performance of the DWELL samples monolithically grown on $\mathrm{Si}$ substrate. The modeling can explain well the dark current performance from $77 \mathrm{~K}$ to $180 \mathrm{~K}$ at relatively high bias, but for the small bias part, the inter-dots carrier transfer process is not includes, which will be further investigated to improve the modeling.

\section{REFERENCES}

[1] C. G. Burguete, D. Guo, P. Jurczak, F. Cui, M. Tang, W. Chen, et al., "Direct growth of InAs/GaSb type II superlattice photodiodes on silicon substrates," IET Optoelectronics, vol. 12, pp. 2-4, 2017.

[2] W. Chen, Z. Deng, D. Guo, Y. Chen, Y. Mazur, Y. Maidaniuk, et al., "Demonstration of InAs/InGaAs/GaAs Quantum Dots-in-a-well Midwave Infrared Photodetectors Grown on Silicon Substrate," Journal of Lightwave Technology, 2018.

[3] L. Lin, H. Zhen, N. Li, W. Lu, Q. Weng, D. Xiong, et al., "Sequential coupling transport for the dark current of quantum dots-in-well infrared photodetectors," Applied physics letters, vol. 97, p. 193511, 2010. 